\title{
Localization of Radiating Sources by an Acoustical Array
}

\author{
Joseph Lardiès ${ }^{\mathrm{a}}$, Hua Ma, Marc Berthillier \\ DMA, Institute FEMTO-ST, UMR-CNRS 6174, Besançon, France
}

Received 15 May 2010, Accepted 15 September 2010

\begin{abstract}
Arrays of sensors are used in many fields to detect signals, to resolve closely spaced targets, to estimate the bearing, the position, the strength and other properties of radiating sources whose signals arrive from different positions. The purpose of the work is described in the paper is to provide estimators which are used to localize acoustical sources by an acoustical array of sensors. These estimators are based on acoustic processing algorithms: the conventional beamforming, the minimum variance and the constrained Capon algorithm. It is shown that these adaptive algorithms can detect radiating acoustical sources which are not detectable by classical weighted arrays of sensors.
\end{abstract}

Key words: Acoustical array, Directivity pattern, Minimum Variance algorithm, Source localization

\section{Introduction}

Arrays of receivers are used in many fields: in radio, radar, sonar, seismic exploration, chemical detection, ultrasonic diagnosis, and so forth, to detect weak signals, to resolve closely spaced targets and to estimate the bearing and other properties of a signal source. The receiving sensors may be any transducers that convert the receiving energy to electrical signals. The type of sensors used to detect these signals differ accordingly: microphones for the acoustic signals, electromagnetic antennas for radio waves, accelerometers/seismometers for the detection of earthquakes, ultrasonic probes and X-ray detectors in medical imaging, containers with membranes or biosensors for gas detection and so forth. In all these highly diverse applications of array signal processing, the sensors are designed with one basic objective in mind: to provide an interface between the environment in which the array is embedded and the signal processing part of the system and the physical manner in which this interface is established depends on the application of interest. In these applications, the goal is to determinate the distribution of the emitted energy in the medium (air, water, rock, etc) that surrounds the array. For example, in industrial environments to localize complex noise fields, in underwater surveillance with sonar systems, in communications to separate speakers, in seismology for the monitoring and analysis of global earthquakes, in all these cases signals received by sensor arrays are processed to obtain estimates of their strength and direction of arrival. In this communication we are concerned in industrial noise localization which has significant effects on labor's health and community living quality. It is highly desired to develop methods that are capable of locating noise sources in an accurate and systematic manner before any noise control measure can be applied. In all that follows, we consider only idealized arrays, those in which there are no cross-couplings between individual receivers. The receivers are assumed to sample the spatial field without distorting it. We further assume that the spatial field in the vicinity of the array is homogenous, an assumption that is often valid when all signal sources are distant from the array, and that all receivers have the same sensitivity.

In this paper we are concerned in industrial noise localization which has significant effects on labor's health and community living quality. It is highly desired to develop methods that are capable of locating noise sources in an accurate and systematic manner before any noise control measure can be applied. Conventional ways of noise source identifications include, for example, sound pressure measurement, sound intensity measurement and acoustic holography. These methods suffer from the drawbacks of being either inaccurate or being restrictive in only small areas or short distances when applied to complex noise fields in industrial environment. An alternative is the use of an array of sensors whose outputs are processed. The performances of these methods in the localization of closely spaced sources are presented. The approach taken here is to assume that the signal field at the array is comprised of $\mathrm{K}$ independent plane wave arrivals from unknown directions and the problem reduces to estimating the $\mathrm{K}$ directions in a background noise environment. One main of the work described in the paper is to provide estimators which are simple to implement on line. These estimators are based on acoustic processing algorithms: the conventional beamforming estimator, the Minimum Variance (MV) estimator and the robust Capon estimator.

\section{Beamforming}

\subsection{Conventional beamformer}

The principles of conventional beamforming were established many decades ago. Consider a linear array as shown in Fig.1. A plane wavefront arriving from a direction normal to the array (broadside array) arrives at all the receivers in phase, and so if summed the signal outputs from the receivers will add in phase and reinforce one another; signals from

\footnotetext{
${ }^{\mathrm{a}}$ Corresponding author: joseph.lardies@univ-fcomte.fr
} 
other directions will not be in phase, and so will not be reinforced. If it is desired to receive narrowband signals from some other direction than broadside, we simple shift the phases of the receiver outputs by appropriate amounts to bring all the receiver outputs into phase before summing. More generally, if the signals are broadband, we time delay the receiver outputs appropriately before summation. This process is called beamsteering. It can readily be seen that the same principles can be extended to arrays of arbitrary geometry in two or three dimensions: we simple insert the appropriate time delays.

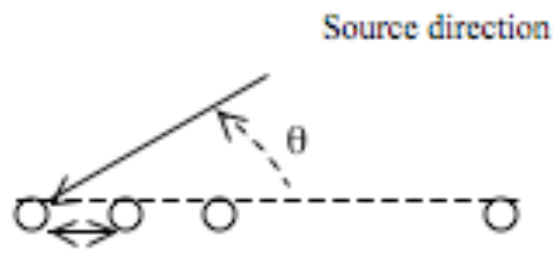

Fig. 1. A linear array of $\mathrm{N}$ elements

We consider a linear array of $\mathrm{N}$ equally spaced elements, located along the horizontal axis, with an element spacing of d. The beamformer output usually called the space or array factor is given by [1]:

$y=\sum_{\mathrm{i}=1}^{\mathrm{N}} a_{\mathrm{i}} v_{\mathrm{i}}\left(k, \theta_{0}\right)=\sum_{\mathrm{i}=1}^{\mathrm{N}} a_{\mathrm{i}} \exp \left[j(i-1) k d\left(\cos \left(\theta-\cos \theta_{0}\right)\right)\right](1)$ where $a_{i}$ are weighting coefficients used to control the shape of the directivity diagram, $\theta_{0}$ determines the direction of the maximum output (the direction of main beam) and $k$ the wavenumber of interest. Note $a$ and $v$ the columns vectors whose entries are $\left\{a_{i}\right\}$ and $\left\{v_{i}\right\}$. Equation (1) may be written as:

$$
y=a^{\mathrm{T}} v=v^{\mathrm{T}} a
$$

where $\mathrm{T}$ is the transpose vector.

In the case of a uniform weighting array we have $\mathrm{a}_{\mathrm{i}}=1$, $\mathrm{i}=1,2, \ldots, \mathrm{N}$ and it can be shown, that in order to avoid grating lobes, the condition for a scanned beam becomes :

$$
d / \lambda \leq \frac{1}{1+\left|\cos \left(\theta_{0}\right)\right|}
$$

If we plot the average power output as a function of signal direction, we obtain the polar diagram or polar response. By convention, the polar response is normalized so its response in the beamsteered direction $\theta_{0}$ is unity. The beamwidth, that is the width of the main lobe, often defined as the angle between the points at which the power response falls by half, indicates the ability to resolve closely-spaced signal sources; the level of the sidelobes is a measure of susceptibility to interference by unwanted signals in directions away from the main beam. The directivity pattern, also known as the polar diagram is obtained by taking the normalized magnitude of $y$ as a function of the polar angle $\theta$ for a given frequency. For a uniform weighting array we have the normalized magnitude:

$$
D=\left|\frac{\sin (N \Psi / 2)}{N \sin (\Psi / 2)}\right|
$$

where $\Psi=k d\left(\cos \theta-\cos \theta_{0}\right)$. For broadside arrays, where the beam maximum occurs in the direction perpendicular to the array axis, we have $\theta_{0}=\pi / 2$. For end-fire arrays where the beam maximum occurs in the direction of the array axis, we have $\theta_{0}=0$. Figures 2 (a) and 2(b) show the antenna directivity diagram with $N=6$ and $d / \lambda=0,4$ for broadside and end-fire arrays. In the case of broadside array, because of rotational symmetry, we are unable to distinguish between signals arriving from $+\pi / 2$ or $-\pi / 2$. We have two beam maximums. In the case of end-fire array we have only a beam maximum.

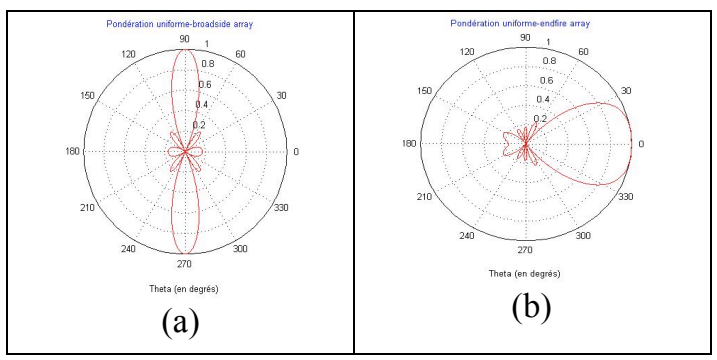

Fig. 2. Directivity diagrams for broadside array (a) and end fire array (b)

It is instructive to examine the behaviour of the directivity diagram. In particular, it can be manipulated to yield information about locations of major maxima or beams, locations of nulls, angular widths of major maxima or beamwidths and locations of minor maxima or sidelobes.

\subsection{Dolph-Tchebychev and binomial beamformer}

Shading is a well-known technique used to modify the polar diagram of an array and low sidelobes are usually desirable to reduce the effect of interfering signals. Dolph [1] addressed that problem by noting the behaviour of the Tchebychev polynomials and devising suitable transformations of variables to link the behaviour of the polynomials to array side lobe levels. With the Dolph-Tchebychev shading the sidelobes all have the same peak level. For an even number of sensors the beamformer output is:

$$
y=2 \sum_{i=1}^{N / 2} a_{i} \cos [(2 i-1) \Psi / 2]
$$

We consider $t=\cos (\Psi / 2)$ and use the Moivre formula to write each $\cos (p \Psi / 2)$ function as a polynomial of degree $p$, called Tchebychev polynomial and noted $\mathrm{T}_{p}(t)$. With this Tchebychev polynomial equation (5) becomes

$$
y=2 \sum_{\mathrm{i}=1}^{N / 2} a_{\mathrm{i}} \mathrm{T}_{2 \mathrm{i}-1}(t)=T_{n-1}\left(t Z_{0}\right)
$$

where $Z_{0}$ is associated to the sidelobes level. By identification we obtain the weighting coefficients $a_{i}$. Figure 3(a) shows the end-fire polar diagram of the Dolph-Tchebychev shading with six elements, $d / \lambda=0,4$ and a constant sidelobe level of $20 \mathrm{~dB}$. The sidelobes all have the same peak level 
which has been selected to be much lower than the levels for the conventional beamformer.

In order to suppress the sidelobes we use weighting coefficients $\mathrm{C}_{N-1}^{p}$ and we obtain for an even number of elements the beamformer output is:

$\mathrm{y}=\sum_{\mathrm{i}=1}^{N / 2} \mathrm{C}_{N-1}^{N / 2-\mathrm{i}} \cos [(2 i-1) \Psi / 2]=2^{N-1} \cos ^{N-1}(\Psi / 2)$

The fundamental property of the binomial weighting concerns the absence of sidelobes in the polar diagram; this is because the directivity function has a general form in $\cos ^{n-1}(\Psi / 2)$ and this function does not oscillate between 0 and $\pi / 2$. Figure 2 (b) shows the directivity end-fire pattern of the binomial shading with six elements and $d / \lambda=0,4$. The sidelobes are absent but the angular width of the main lobe has increased.

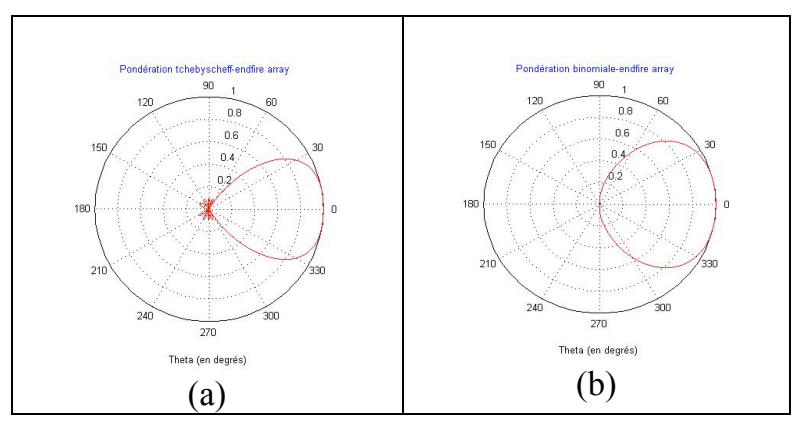

Fig. 3. Directivity diagrams for Dolph-Tchebychev (a) and binomial (b) weighting

\subsection{Superdirectivity and optimal beamforming}

It is sometimes desirable to use a given array at low frequencies. Conventional beamforming then yields a broad beam. However, by appropriate choice of the weight vector $a$, it is possible to generate narrow beamwidths and the array is called superdirective. But there is a penalty: as the frequency is reduced, the magnitude of the weight required becomes large, making the practical implementation of the superdirectivity difficult. Schelkunoff [2] showed that every linear array with commensurable separations between the elements can be represented by a polynomial and every polynomial can be interpreted as a linear array. The analytical representation of an output array is accomplished with the aid of the following transformation: $\mathrm{z}=\exp (\mathrm{j} \Psi)$ and we obtain :

$$
y=\sum_{i=0}^{N-1} a_{i} z^{i}
$$

This polynomial can be written in terms of its (n-1) roots $\left(z_{1}, z_{2}, \ldots, z_{N-1}\right)$ and Schelkunoff has shown that if these roots are regularly spaced between 0 et $-k d\left(1+\cos \theta_{0}\right)$, the visible range of $z$, we have a superdirective array. Figure 4(a) shows the end-fire directivity pattern with a Schelkunoff distribution, six elements and $d / \lambda=0,4$. The beamwidth, or angular space between the half-power points on each side of the main lobe, is narrower than that of a uniform, DolphTchebychev or binomial weighted end-fire array.

We address now the problem of selecting a set of weights which maximize the directivity index of a passive acoustical array. The directivity index is defined as:

$$
D(f)=\frac{\left|y\left(f, \Omega_{0}\right)\right|^{2}}{\frac{1}{4 \pi} \int(\Omega)|y(f, \Omega)|^{2} \mathrm{~d} \Omega}=\frac{a^{* \mathrm{~T}} V\left(f, \Omega_{0}\right) a}{a^{* \mathrm{~T}} W(f) a}
$$

where $\left|y\left(f, \Omega_{0}\right)\right|^{2}=a^{* \mathrm{~T}} V\left(f \Omega_{0}\right) a$ is the power out of the beamformer steered in direction implied by $\Omega_{0}$, or in the direction of the main beam, and $\frac{1}{4 \pi} \int_{(\Omega)}|y(f, \Omega)|^{2} \mathrm{~d} \Omega=$ $a^{* \mathrm{~T}} \mathrm{Wa}$ the average output power.

In these expressions we have : $V\left(f, \Omega_{0}\right)=v^{*}\left(f, \Omega_{0}\right) v^{\mathrm{T}}\left(f, \Omega_{0}\right)$ and $W_{i j}=\frac{1}{4 \pi} \int_{(\Omega)} v_{i}^{*} v_{j} \mathrm{~d} \Omega$. The denominator of (9) is the output power when the acoustical array is in a far field of plane waves arriving from all directions and having the same level. This is also the definition of the omnidirectional noise. In fact, the directivity index represents also the output signalto-noise ratio which may be defined as the ratio of the power received per unit solid angle in the direction of the signal to the average noise power received per unit solid angle. The purpose is to determine an optimum set of weights $\left\{a_{i}\right\}$ in amplitude and phase to yield the maximum directivity index with respect to a prespecified number of elements, spacing and frequency. The index directivity is expressed as the ration of two quadratic forms and it is shown [3] that maximisation of (9) is obtained when: $a=W^{1} v$ and the directivity index is : $D_{\operatorname{Max}}(f)=v^{* \mathrm{~T}} W^{1} v$. Figure 4(b) shows the pattern of the six-element end-fire array with $d / \lambda=0,4$ yielding maximum directivity index.

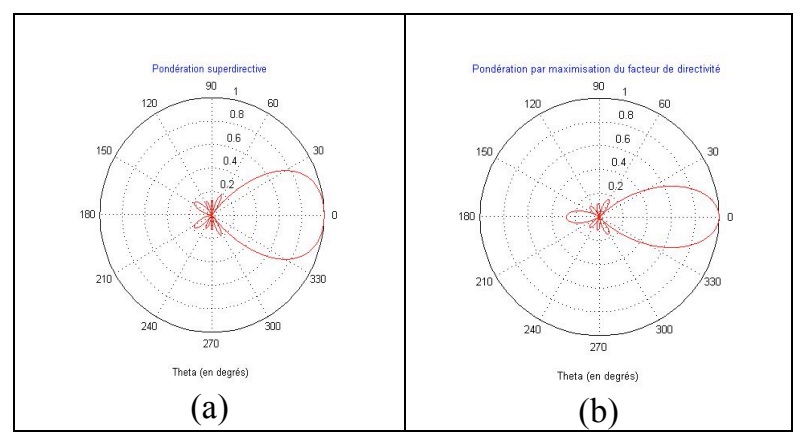

Figure 4. Directivity diagrams for superdirective (a) and optimal (b) weighting

A comparison of different weighting techniques developed in the communication are presented in table 1 for a linear endfire array of six elements and a value of $d / \lambda=0,4$. 
Table 1. Comparison of weighting methods

\begin{tabular}{|l|c|c|c|c|c|}
\hline weighting & Uniform & Dolph & Binomial & Schelk & optimal \\
\hline beamwidth & $84^{\circ}$ & $90^{\circ}$ & $126^{\circ}$ & $78^{\circ}$ & $58^{\circ}$ \\
\hline sidelobes & Important & low & absent & low & low \\
\hline
\end{tabular}

However, these weighted arrays have a fundamental resolution problem in the localization of different sources. Figure 5 shows the performances of the optimal end-fire array in the localisation of two sources at $10^{\circ}$ and $19^{\circ}$ relative to the antenna axis. An important beamwidth and important sidelobes are clearly present in this spectra and it is impossible to obtain the direction of arrival of these two sources.

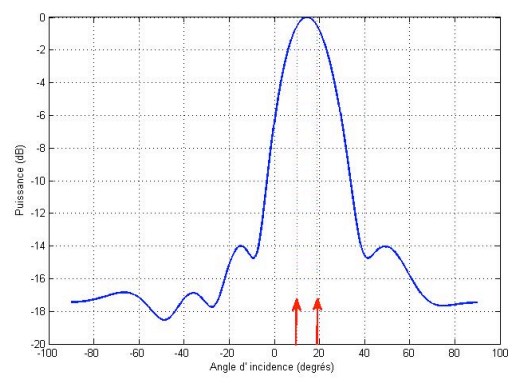

Fig. 5. Direction of arrival of two sources using the optimal beamforming

\section{Adaptive array}

\subsection{Spatial correlation matrix}

The receiving array has $\mathrm{N}$ omnidirectional sensors and is immersed in an acoustic noise field which consists of $\mathrm{K}$ independent discrete sources. Because of the geometric positions of the sensors, the total signal power incident on each sensor is the same, but the phase information is different on each receiver.

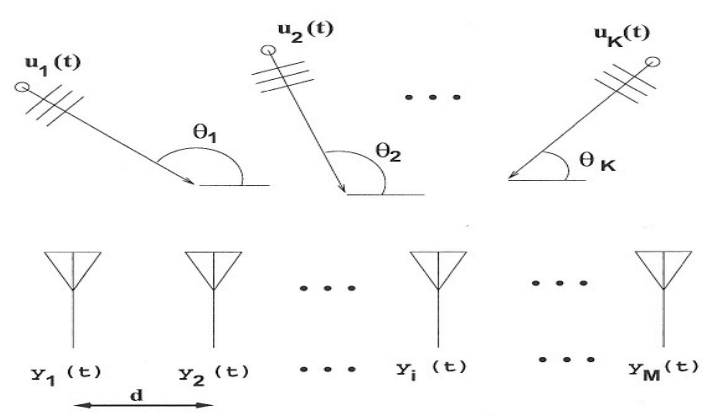

Fig.6. The setup of the source location problem

The purpose of any estimator is to use the phase information in some way to infer which signals reached the receiving array and the goal of sensor array source localization is to find the locations of sources of wavefields that impinge on the acoustical array. The available information is the geometry of the array, the parameters of the medium where wavefields propagate and the time measurements or outputs of the sensors. For purposes of exposition, we first focus on the narrowband scenario. For a set of $\mathrm{K}$ sources, the signals observed at the outputs of the $\mathrm{N}$ sensors array are represented by the N-dimensional vector [4-6]

$$
x(t)=\sum_{i=1}^{\mathrm{K}} b\left(\theta_{i}\right) s_{i}(t)+n(t)
$$

where $s_{i}(t)$ is the complex amplitude of the $\mathrm{i}^{\text {th }}$ source. It is a zero-mean complex random variable $: \mathrm{E}\left[s_{i}(t)\right]=0$. The signal power $p_{i}$ of the $\mathrm{i}^{\text {th }}$ source which we wish to localize is represented by its variance $p_{i}=\operatorname{Var}\left[s_{i}(t)\right]=\mathrm{E}\left[s_{i}(t) s_{i}(t)^{*}\right]$. Here E[ ] denotes an ensemble average and the superscript* represents the complex conjugate. The direction of arrival of the $i^{\text {th }}$ signal is represented by the $\mathrm{N}$-dimensional vector $b\left(\theta_{i}\right)=\left[b_{1}\left(\theta_{i}\right) b_{2}\left(\theta_{i}\right) \ldots b_{N}\left(\theta_{i}\right)\right]^{\mathrm{T}}$, often called the array manifold vector or the steering vector or the directional mode vector and $n(t)$ is the additive noise. The noise is assumed to be spatially white (independent or uncorrelated from sensor to sensor) and the same power level of noise is present in each receiver. With these assumptions, the cross-spectral matrix for the noise alone is $R_{N}=E\left[n(t) n(t)^{H}\right]=p_{N} I$ where $p_{N}$ is the noise power, $I$ is the $(\mathrm{NxN})$ identity matrix and the superscript $H$ denotes the complex-conjugate transpose operation. Equation (10) may be rewritten in the matrix form

$$
x(t)=B s(t)+n(t) \quad \mathrm{t} \in\left\{\mathrm{t}_{1}, \mathrm{t}_{2}, \ldots \mathrm{t}_{\mathrm{N}}\right\}
$$

The (NxK) matrix $B$ where each column is a source direction vector, is the so-called array manifold matrix. For any single plane wave arrival, the outputs from the $\mathrm{N}$ individual receivers will differ in phase by an amount determined by the geometry of the array and the arrival direction. In other words, the elements $B_{q r}$ of the matrix $B$ are functions of the signal arrival angles and the array elements locations. Thus, one has $B_{q r}=\exp \left(\mathrm{j} \phi_{\mathrm{qr}}\right)$ where $\phi_{\mathrm{qr}}$ is the phase of the signal at the $\mathrm{q}^{\text {th }}$ receiver from the $\mathrm{r}^{\text {th }}$ source, measured relative to some arbitrary reference point. That is, $B_{q r}$ depends on the $\mathrm{q}^{\text {th }}$ array element, its position relative to the origin of the coordinate system, and its response to a signal incident from the direction of the $\mathrm{r}^{\text {th }}$ source. $s(t)$ is the K-dimensional vector, the components of which are the complex amplitudes of the sources. It can readily be seen that the output signal from the $\mathrm{q}^{\text {th }}$ sensor may be written as :

$$
x_{q}(t)=\sum_{\mathrm{r}=1}^{\mathrm{K}} B_{q r}(\theta) s_{r}(t)+n_{q}(t)
$$

Since the $\mathrm{K}$ arrivals are by assumption independent, the correlation matrix between the different signal sources is

$$
R_{S}=E\left[s(t) s(t)^{H}\right]=\operatorname{diag}\left(p_{1}, p_{2}, \ldots, p_{K}\right)
$$

and at the operating frequency, the spatial correlation matrix (or covariance matrix) of the receiver outputs may be expressed, for signals uncorrelated of each other and of noise, as

$$
R=E\left[x(t) x(t)^{H}\right]=B R_{S} B^{H}+R_{N}
$$

In practice, the spatial correlation matrix is estimated by a finite number of time domain samples (snapshots) and the following estimated form is used : 


$$
\hat{R}=\frac{1}{M} \sum_{i=1}^{\mathrm{M}} x\left(t_{i}\right) x\left(t_{i}\right)^{H}
$$

where $x\left(t_{i}\right)$ is the array signal vector sampled at time $t_{\mathrm{i}}$ and $M$ is the number of such samples. The caret $\left(^{\wedge}\right)$ denotes an estimated value.

We can now derive estimators for source localization using an acoustical array.

\subsection{The minimum variance estimator}

The minimum variance (MV) estimator was originally proposed by Capon who conducted a frequency wavenumber analysis on earthquake data analysis. The conventional beamformer can be considered as a kind of linear spatial filter with data-independent coefficients. In contrast, the minimum variance method (or Capon's method) can be considered as a kind of data-dependent spatial filter, in which the coefficients are chosen such that the filter has constant gain at a particular direction while its output power is minimized. The underlying principle of the method amounts to finding an optimal steering vector $w_{\text {opt }}$ such that the array output power is minimized while maintaining the gain along the look direction to be constant, say unity [4]. That is,

$$
\begin{array}{r}
\min w^{H} R w \\
\text { subject } \quad w^{H} b(\theta)=1
\end{array}
$$

Minimizing the resulting beam energy reduces the contributions to this energy from sources and noise not propagating in the direction of look. The solution of this constrained optimization problem occurs often in the derivation of adaptive array processing algorithms. The solution technique is to use a Lagrange multiplier $\lambda$ and a cost function

$$
H(w)=w^{H} R w+2 \lambda\left(w^{H} b(\theta)-1\right)
$$

The gradient with respect to $w$ is

$$
\operatorname{grad} H(w)=2 R w+2 \lambda b(\theta)
$$

and the minimum of the cost function is obtained when

$$
w_{\text {opt }}=-\lambda R^{-1} b(\theta)
$$

But $w_{\text {opt }}$ must satisfy the constraint, one then has

$$
\begin{gathered}
\lambda=\left(-b^{H}(\theta) R^{-1} b(\theta)\right)^{-1} \\
w_{\text {opt }}=R^{-1} b(\theta)\left(b^{H}(\theta) R^{-1} b(\theta)\right)^{-1}
\end{gathered}
$$

and the corresponding array output power is

$$
p_{M V}(\theta)=E\left[|\mathrm{y}(\mathrm{t})|^{2}\right]=\left(b^{H}(\theta) R^{-1} b(\theta)\right)^{-1}
$$

The goal of the minimum variance estimator is that the contributions of the signals from directions other than $\theta$ to the array output are minimized while the signal at direction $\theta$ passes through without any distortion. Equation (23) is also known as the angular power spectrum of the minimum variance estimator and the signal directions are found by the locations of the spectrum peaks. The peaks level of the spectrum give a good estimate of the true targets power and the spectrum also has uniformly low sidelobes. Simulations have shown that this estimator gives satisfactory resolution if the number of snapshots is high. The algorithm does not require any knowledge of the number of sources present and can also be used with irregular arrays. It is expected that this estimator performs better than the classical beamforming and has super-resolution provided that the SNR is moderately high, the sources are not strongly correlated and the number of snapshots is sufficient.

The common advantage of this estimator is that it does not assume anything about the statistical properties of the data and, therefore, it can be used in situations where we lack information about these properties. Figure 7 shows the power spectral density of the adaptive array with six sensors and $d / \lambda=0,4$. Two sources at $0^{\circ}$ and $20^{\circ}$ relative to the antenna axis are present are localized with this estimator.

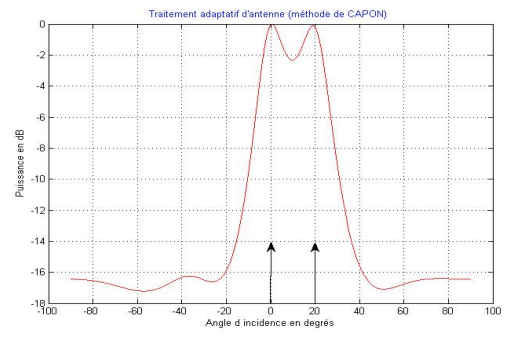

Fig. 7. Direction of arrival of two sources using the adaptive estimator

The resolution of the antenna array can be improved using a robust adaptive array.

\subsection{The robust minimum variance estimator}

The minimum variance estimator adaptively selects the weight vector to minimize the array output power subject to the linear constraint that the signal of interest does not suffer from any distortion. This beamformer has a better resolution and much better interference rejection capability than the standard beamformer, provided that the array steering vector corresponding to the signal of interest is accurately known. However, the knowledge of the signal of interest steering vector can be imprecise, which is often the case in practice due to the differences between the assumed signal arrival angle and the true arrival angle. Whenever this happens, the minimum variance estimator can give poor results. A robust minimum variance estimator is then proposed based on a diagonal loading [7] where a scaled identity matrix is added to the covariance matrix given in (14). The main idea of diagonal loading is to replace the covariance matrix $R$ by the matrix $R+r I$ where the diagonal-loading factor $r$ is a userselected parameter. The weight vector so obtained is given by :

$$
w=(R+r I)^{-1} b(\theta)\left[b^{* T}(\theta)(R+r I)^{-1} b(\theta)\right]^{-1}
$$

The robust minimum variance power estimate of array output is then :

$$
P_{R M V}=\left[b^{* T}(R+r I)^{-1} R(R+r I)^{-1} b\right]\left[b^{* T}(R+r I)^{-1} b\right]^{-2}
$$

Figure 8 shows the power spectral density of the robust adaptive array with six sensors and $d / \lambda=0,4$. Two sources at $0^{\circ}$ and $20^{\circ}$ relative to the antenna axis are present are localized with this estimator which has a better resolution than the minimum variance estimator. 


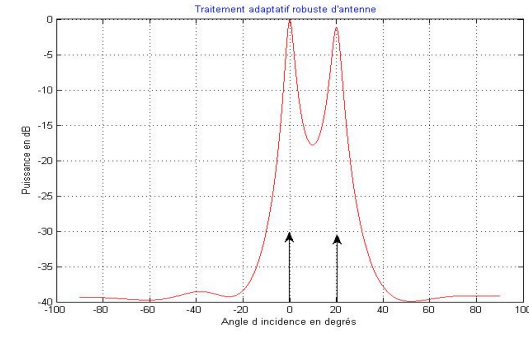

Fig. 8. Direction of arrival of two sources using the robust adaptive estimator

\section{Conclusion}

Beamforming techniques are useful for focusing sound waves but are not adapted for the localization of multiple sources. Two methods using adaptive arrays have been proposed and are based on the minimum variance and the robust minimum variance estimation. These algorithms have been tested by computer simulation studies and they perform satisfactory. In the future the resolution capability of these algorithms and their limitations in a broadband environment will be studied.

\section{References}

1. R. Elliot, Antenna theory, J. Wiley (2003)

2. S.A. Schelkunoff, A mathematical theory of linear arrays, Bell System Technology Journal, 22, 80-107, (1943).

3. F.R. Gantmacher, The theory of matrices, Dunod (1966)

4. P. Stoica, R. Moses, Introduction to spectral analysis, Prentice Hall (1997)

5. J. Lardiès J., Power estimation of multiple sources by an acoustical array, Journal of Sound and Vibration, 145, 309-320, (1991).

6. J. Lardiès, H. Ma, M. Berthillier, E. Foltête, Performance of high-resolution sensor array processing algorithms in the localisation of acoustic sources, Acoustics'08, Paris, June29-July 4, 2008.

7. J. Li, P. Stoica, Z. Wang, On robust Capon beamforming and diagonal loading, IEEE Trans. Signal Processing, 51, 1702-1711, (2003). 